\title{
Erratum to: Maternal parenting styles, homework help, and children's literacy development in language minority and Finnish-speaking families
}

\author{
Riitta Sikiö $^{1}$ • Martti Siekkinen ${ }^{1}$ - Leena Holopainen ${ }^{1}$. \\ Gintautas Silinskas $^{2}$ - Marja-Kristiina Lerkkanen ${ }^{3}$. \\ Jari-Erik Nurmi ${ }^{2}$
}

Published online: 4 October 2017

(C) Instituto Superior de Psicologia Aplicada, Lisboa, Portugal and Springer Science+Business Media B.V. 2017

\section{Erratum to: Eur J Psychol Educ \\ https://doi.org/10.1007/s10212-017-0330-4}

In the original publication, some data were published incorrectly. The authors apologize for the errors. Below are the corrections:

\begin{abstract}
:
Sentence found in lines 6-7 should be rewritten as: "Furthermore, the more LM mothers used warmth and psychological styles of parenting, the more they helped their daughters..."

Sentence found in line 9 should be rewritten as: LM maternal behavioral control parenting styles were positively related, and psychological control was negatively related to children's reading skills through help with homework.

This sentence found in lines 11-13 should be deleted: This research shows that warmth and behavioral parenting styles with positive educational atmosphere helps children to get better literacy achievement in school.
\end{abstract}

The online version of the original article can be found at https://doi.org/10.1007/s10212-017-0330-4

\section{Riitta Sikiö}

riitta.sikio@edu.parikkala.fi

1 Philosophical Faculty, University of Eastern Finland, P.O. Box 111, FI-80101 Joensuu, Finland

2 Department of Psychology, University of Jyväskylä, Jyväskylä, Finland

3 Department of Teacher Education, University of Jyväskylä, Jyväskylä, Finland 


\section{Mothers' parenting styles and maternal help in the language minority (LM) group}

Page 8, first paragraph, lines 3-4, sentence should be rewritten as: Results (Fig. 1) showed that mothers' warmth and psychological parenting styles correlated positively and behavioral control negatively to helping girls.

Page 8, second paragraph should be rewritten as: The results also showed a significant positive indirect relation of LM mothers' behavioral control on reading skills $(0.35, p<.01)$ and decoding skills $(0.46, p<.01)$ with maternal help. There was also a significant negative indirect relation of mothers' psychological control on reading comprehension skills $(-0.41, p<$ $.01)$ and decoding skills with maternal help $(-0.56, p<.001)$.

\section{Discussion}

Page 10, Paragraph 3 should be rewritten as: The result confirms earlier research that demonstrated the positive relations of relative warm and authoritative parenting with moderate control, which also offers a positive outcome in school achievement (Steinberg et al. 1989; Cox and Paley 2003; Landry et al. 2006; Fletcher et al. 2008; Newman et al. 2015). The parenting style of LM mothers can be explained in terms of warm motherdaughter relationships and mothers' trust in their children's abilities (see Sikiö et al. 2016), which strengthen intrinsic motivation. This positive cycle enables a child's success with literacy.

Paragraph 5, should be rewritten as: In this study, LM group children comprised 18 different countries, and their parenting styles differed from Finnish parenting styles, indicating the relations of different cultures on parenting styles. The explanation for different parenting styles could also be found in cultural differences surrounding raising children. Mothers' sensitivity to their children's learning Finnish and its difficulties could cause them to exercise more psychological control. Like the LM mothers in this study, mothers who cannot speak native Finnish properly have more difficulty helping their children with Finnish literacy tasks (Aram 2007). Different parenting styles may also be related to acculturation (Prady et al. 2013). The new home country, in this case Finland, could offer a limited or even poor culture that families do not want to identify with. This could influence both LM parents' knowledge of the MP school system and their ability to help with homework. For example, teachers give daily homework assignments to their pupils according to grade level. Homework is not required for preschool, but first and second grades usually require $30 \mathrm{~min}$ to $1 \mathrm{~h}$ of homework each day and 1 to $2 \mathrm{~h}$ for a child in third or fourth grade. Most homework tasks are designed to reinforce what has been taught in class, but in the upper grades, children are increasingly expected to acquire additional information at home by means such as the internet. Parents with insufficient knowledge of the Finnish language or educational system may experience difficulties in helping their children.

Page 11, seventh paragraph, first sentence should be rewritten as: The results of maternal help regarding children's academic skills showed a negative impact on reading comprehension and decoding skills for both groups, and on the pseudoword spelling test for the MP group.

Conclusions should be rewritten: Mothers in the LM group used significantly more psychological control in their parenting than did MP mothers. Behavioral control influenced positively and psychological control negatively indirectly through the maternal help to reading 
skills in the LM group. The higher the mother's education, the less they helped their daughters. This study opens up a new way of thinking about parenting styles and their different influences on school success.

P. 12, lines 1-2 should be rewritten as: Behavioral control influenced positively and psychological control negatively indirectly through the maternal help to reading skills in the LM group.

P. 12, deleted sentence: This research reveals the idea that warmth and the behavioral control style of parenting have a positive impact on children's academic skills.

\section{Figures and tables}

Figure 1, the following should be inserted in the caption:

Psychological control $><$ Maternal help .93, Behavioral control $><$ Maternal help -.79 , Maternal help $><$ Reading comprehension (girls) -.43 , Warmth $><$ Maternal help .34

Table 2, the following should be inserted in the table footnote:

LM decoding $><$ Maternal help -.54 , Pseudoword spelling $><$ Maternal help .02

Decoding $><$ Psykol control .18, Pseudoword spelling $><$ Psykol control-.12

Decoding $><$ Warmth -.03 , Pseudoword spelling $>$ Warmth.03

Decoding $><$ Behavioral control .24, Pseudoword spelling $><$ Behavioral control- .17

MP decoding $><$ Behavioral control .04, Pseudoword spelling $><$ Behavioral control .02

The original article was corrected. 